\title{
EXAFS study of the amorphous phase of InP after swift heavy ion irradiation
}

\author{
C.S. Schnohr ${ }^{\text {a,* }}$, P. Kluth ${ }^{\text {a }}$, A.P. Byrne ${ }^{\text {b,c }}$, G.J. Foran ${ }^{\text {d }}$, M.C. Ridgway ${ }^{\text {a }}$ \\ ${ }^{a}$ Department of Electronic Materials Engineering, Research School of Physical Sciences and Engineering, \\ Australian National University, ACT 0200, Australia \\ ${ }^{\mathrm{b}}$ Department of Nuclear Physics, Research School of Physical Sciences and Engineering, Australian National University, ACT 0200, Australia \\ ${ }^{\mathrm{c}}$ Department of Physics, Faculty of Science, Australian National University, ACT 0200, Australia \\ d Australian Nuclear Science and Technology Organisation, Menai, Australia
}

Available online 10 January 2007

\begin{abstract}
Structural parameters of InP amorphised by electronic energy deposition were determined using extended X-ray absorption fine structure spectrometry. Samples were prepared with $180 \mathrm{MeV} \mathrm{Au}{ }^{13+}$ irradiation and a combination of semiconductor processing techniques and chemical etching. At the In $K$-edge of the amorphous material, only the first shell scattering peak is observed demonstrating the structural disorder introduced by the swift heavy ion irradiation. Furthermore, the presence of chemical disorder in the form of InIn bonds is shown. These homopolar bonds amount to $10 \%$ of the total number of In coordinations which is $3.73 \pm 0.41$ and thus less than the crystalline value of 4 . In general, these results are similar to those reported for InP and other compound semiconductors amorphised by conventional ion implantation with a dominant nuclear energy deposition. However, slight differences in the values of the structural parameters obtained remain and further studies are necessary to determine their origin.
\end{abstract}

(C) 2007 Elsevier B.V. All rights reserved.

PACS: 61.10.Ht; 61.43.Dq; 61.80.Jh

Keywords: InP; Amorphous structure; Extended X-ray absorption fine structure spectrometry; Swift heavy ion irradiation

\section{Introduction}

Amorphous semiconductors and their atomic-scale structure are of technological as well as scientific interest and have been studied with techniques such as extended X-ray absorption fine structure (EXAFS) spectroscopy [1]. Among the compound semiconductors, InP is a very promising material for various electronic applications. Its amorphisation behaviour due to conventional ion implantation has been investigated over a wide range of ion species, energies and irradiation temperatures [2]. EXAFS studies of the structure of the amorphous phase produced by ion implantation report not only increased structural disorder compared to the crystalline material but also the

\footnotetext{
${ }^{*}$ Corresponding author. Tel.: +612612 50374; fax: +61261250511.

E-mail address: css109@rsphysse.anu.edu.au (C.S. Schnohr).
}

presence of chemical disorder in the form of homopolar bonding $[3,4]$. These so-called "wrong" bonds are believed to play a key role in the amorphisation process of III-Vsemiconductors as suggested by first-principles moleculardynamics (MD) simulations of amorphous InP produced by rapid quenching from the liquid [5]. In the framework of the thermal spike model [6] it is believed that for some materials the irradiation with swift heavy ions (SHI) produces a melt around the ion trajectory which when quenched fast enough leads to the formation of amorphous tracks. For this to happen the electronic energy loss of the incoming ion has to exceed a certain threshold value. The formation of such amorphous tracks and complete amorphisation at higher ion fluences was first reported for InP by Herre et al. [7]. Since then SHI irradiation of InP has been studied due to its scientific and technological implications [8], however, no investigation of structural 


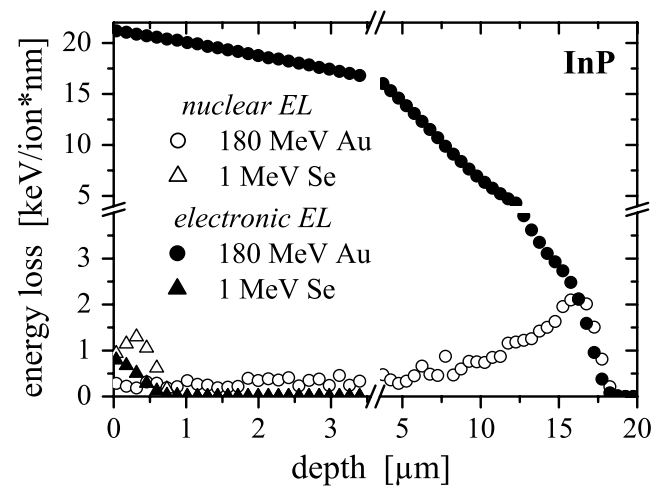

Fig. 1. Nuclear and electronic energy loss as a function of depth as calculated by SRIM2003 [9] for InP irradiated with $1 \mathrm{MeV} \mathrm{Se}$ and $180 \mathrm{MeV}$ Au.

parameters has been reported. As apparent from Fig. 1, the depth regions of dominant electronic and nuclear energy loss are well separated for ions in the energy range of several hundred $\mathrm{MeV}$ leading to the formation of two distinct amorphous layers [8]. Gaiduk et al. [10] have found from transmission electron diffraction studies that the shortrange order of these two amorphous layers is different depending on the dominant form of energy loss. For the current report, original experiments were performed to determine the structure of InP amorphised by electronic energy deposition using EXAFS spectrometry.

\section{Experimental details}

In order to separate the regions of dominant electronic and nuclear energy loss of the SHI irradiation an InP/ $\mathrm{In}_{0.53} \mathrm{Ga}_{0.47} \mathrm{As} / \mathrm{InP}$ heterostructure $\left[2.75 \mu \mathrm{m} / 50 \mathrm{~nm} /\left(\begin{array}{lll}1 & 0 & 0\end{array}\right)\right.$ substrate] was grown by metal organic chemical vapour deposition. These samples were irradiated at room temperature with $180 \mathrm{MeV} \mathrm{Au}{ }^{13+}$ ions to a fluence of $3 \times 10^{13} \mathrm{~cm}^{-2}$ using the 14UD Heavy-Ion Accelerator at the Australian National University, Canberra. A beam scanning system was applied to homogeneously irradiate a sample area of $3 \times 5 \mathrm{~mm}$. The samples were mounted on the sample holder with carbon paste to facilitate good thermal contact and the flux was kept between $1.5-2.5 \times 10^{10} \mathrm{~cm}^{-2} \mathrm{~s}^{-1}$. The chosen ion fluence is sufficient to produce an extended amorphous layer, as confirmed by Rutherford backscattering spectrometry (RBS) measurements in channelling configuration. Fig. 2 shows the energy spectra of $2 \mathrm{MeV}$ He ions backscattered under an angle of $\vartheta=168^{\circ}$. It can be seen that the aligned yield of the sample irradiated with $3 \times 10^{13} \mathrm{Au} \mathrm{cm}{ }^{-2}$ matches the yield in random direction demonstrating the amorphous nature of the material. After SHI irradiation the InP surface layer was lifted off using a chemical process similar to the one described in [3]. The amorphised InP surface layer was masked with Apeizon black wax. Then the InP substrate was removed by etching in $\mathrm{HCl}(37 \%): \mathrm{H}_{2} \mathrm{O}(5: 1)$ for $\sim 1 \mathrm{~h} 20 \mathrm{~min}$ using the $\mathrm{In}_{0.53} \mathrm{Ga}_{0.47} \mathrm{As}$ as a selective etch stop. The small In content

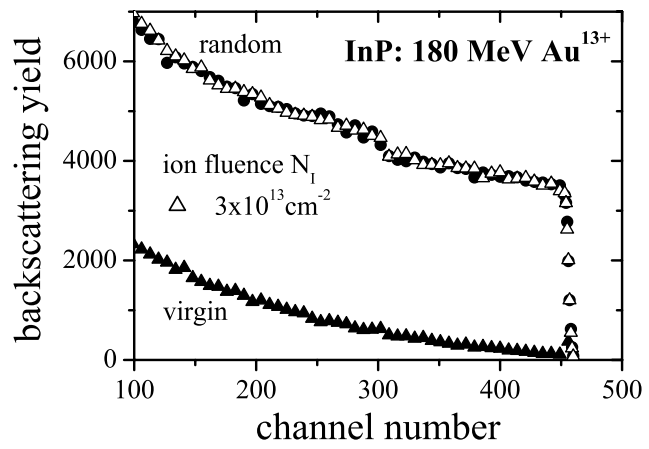

Fig. 2. RBS channelling spectra of $2 \mathrm{MeV} \mathrm{He}$ ions backscattered on (100)-InP prior to and after irradiation with $180 \mathrm{MeV} \mathrm{Au}^{13+}$ at room temperature.

present in the $\mathrm{In}_{0.53} \mathrm{Ga}_{0.47} \mathrm{As}$ layer does not effect the EXAFS signal as confirmed for crystalline samples prepared in the same way. The amorphised InP films were then mounted on Kapton tape and the black wax was removed using trichloroethylene. The whole process is illustrated in Fig. 3. For the EXAFS measurements two such films were mounted together giving a total sample thickness of $\sim 5.5 \mu \mathrm{m}$. This method of sample preparation is free of artifacts [1] and ensures that the structure investigated with the EXAFS measurements was produced by the electronic energy loss of the SHI and that the contribution of the nuclear energy deposition can be neglected (compare Fig. 1). A crystalline standard was prepared with the same method of selective etching of the $\operatorname{In}_{0.53} \mathrm{Ga}_{0.47} \mathrm{As} / \mathrm{InP}$ heterostructure without prior ion irradiation.

EXAFS measurements were carried out at the Australian National Beamline Facility at the Photon Factory in Japan approximately four weeks after SHI irradiation. Measurements were performed at the In $K$-edge in fluorescence mode at a temperature of $\sim 12 \mathrm{~K}$. The data was processed and fitted using the IFEFFIT code [11] and the corresponding user interfaces ATHENA and ARTEMIS [12]. Phase shifts and backscattering amplitudes were calculated $a b$ initio by FEFF6 [13]. Fittings were performed in radial space in the range of $R=1.6-4.8 \AA$ after Fourier transformation over a photoelectron momentum $(k)$ range of $k=3.8-15 \AA^{-1}$ applying a $k$-weight of three. The amplitude reduction factor $\left(S_{0}^{2}\right)$

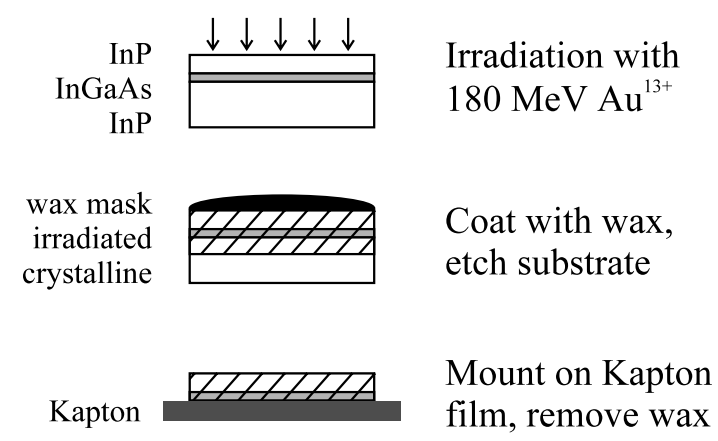

Fig. 3. Schematic diagram of the preparation process utilized to produce EXAFS samples of InP amorphised by electronic energy deposition. 
was determined from the crystalline standard and then kept constant for the amorphous sample.

\section{Results and discussion}

Fig. 4(a) shows the $k^{3}$-weighted EXAFS as a function of $k$ for the crystalline standard and the sample amorphised by SHI irradiation. The EXAFS spectrum of the crystalline material exhibits a complex structure indicating the presence of several different frequencies. On the contrary, the spectrum of the amorphous material seems to be dominated by a single frequency. This picture is confirmed when looking at the Fourier transforms of the EXAFS data shown in Fig. 4(b). For the crystalline standard three different peaks can be distinguished in the range of $R=1-5 \AA$ corresponding to scattering from the first $(\mathrm{P})$, second (In) and third $(\mathrm{P})$ nearest neighbours. For the amorphous sample only the first shell peak is observed. Hence, no coherent scattering is apparent beyond the first shell for samples irradiated with SHI to fluences above the amorphisation threshold. This finding is in good agreement with the results reported by Glover et al. [3] and Azevedo et al. [4] for implantation of InP with conventional ion energies (up to $6.8 \mathrm{MeV} \mathrm{Ge}$ and $8.5 \mathrm{MeV} \mathrm{P}$, respectively) and with the findings for semiconductors amorphised by ion implantation in general [1].
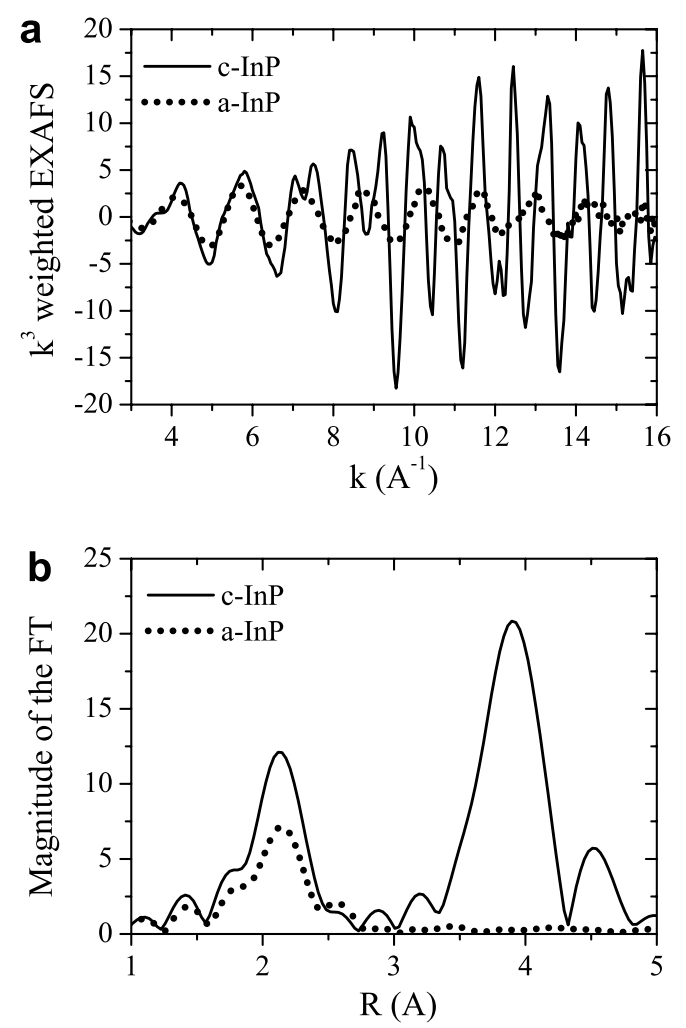

Fig. 4. (a) $k^{3}$-weighted EXAFS spectra of crystalline (c-InP) and amorphous (a-InP) InP (solid and dotted line, respectively) versus the photoelectron momentum $k$. The amorphous phase was produced by SHI irradiation with $180 \mathrm{MeV} \mathrm{Au}{ }^{13}$ ions to a fluence of $3 \times 10^{13} \mathrm{~cm}^{-2}$. (b) Fourier transforms of the spectra shown in panel (a) as a function of radial distance $R$ from the absorber.
A detailed analysis of the EXAFS spectrum of the amorphous sample (see Fig. 4(a)) reveals beats in the amplitude indicating that the first shell data is the superposition of two close but different frequencies. This is consistent with the presence of both In-P and In-In bonds in the amorphous phase. Fig. 5(a) shows the $k^{3}$-weighted EXAFS signal as a function of $k$ for the back-Fourier transformed experimental data and the best fit. The corresponding Fourier transforms are plotted in Fig. 5(b). The latter also shows the two contributions arising from scattering at first nearest neighbour In or $\mathrm{P}$ atoms. Coordination numbers $(\mathrm{CN})$, bond lengths (BL) and Debye-Waller factors (DWF) obtained from the fitting are summarized in Table 1 for the crystalline and the amorphous material. The phase transition due to SHI irradiation leads to a decreased In-P coordination number of $3.36 \pm 0.25$ compared to the crystalline value of 4 and an increase of the DWF to $0.0049 \pm 0.0005 \AA^{2}$ compared to $0.0023 \pm 0.0004 \AA^{2}$ for the In-P bonds of the crystalline standard. These changes are similar to those reported for samples amorphised by conventional ion energies [3,4]. However, an increase of the In-P bond length by $\approx 0.02 \AA$ found in both these works is not seen in the present case. The number of InIn coordinations amounts to $0.37 \pm 0.16$ with an average
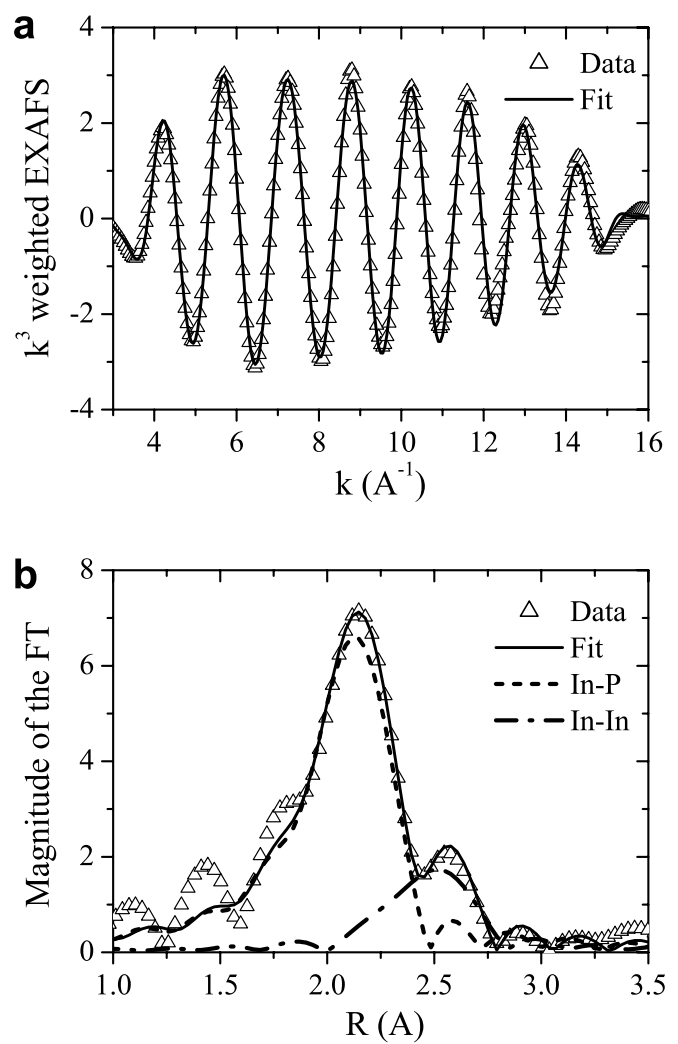

Fig. 5. (a) $k^{3}$-weighted EXAFS spectra as a function of $k$ for the backFourier transformed experimental data of the InP amorphised by SHI irradiation (triangle) and fitting result (solid line). (b) Corresponding Fourier transforms plotted versus $R$. Also shown are the two contributions due to In-P and In-In bonds in the first shell (short-dashed and dash-dotted line, respectively). 
Table 1

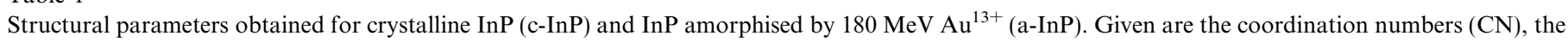
bond lengths (BL) and the Debye-Waller factors (DWF) for the first shell In-P and In-In bonds

\begin{tabular}{|c|c|c|c|c|c|c|}
\hline & \multicolumn{3}{|l|}{ In-P } & \multicolumn{3}{|l|}{ In-In } \\
\hline & $\mathrm{CN}$ & $\mathrm{BL}(\AA)$ & $\overline{D W F}\left(\AA^{2}\right)$ & $\mathrm{CN}$ & $\mathrm{BL}(\AA)$ & DWF $\left(\AA^{2}\right)$ \\
\hline $\mathrm{c}-\mathrm{In} \mathrm{P}$ & 4 & $\begin{array}{l}2.528 \\
\pm 0.004\end{array}$ & $\begin{array}{l}0.0023 \\
\pm 0.0004\end{array}$ & & & \\
\hline a-InP & $\begin{array}{l}3.36 \\
\pm 0.25\end{array}$ & $\begin{array}{l}2.531 \\
\pm 0.003\end{array}$ & $\begin{array}{l}0.0049 \\
\pm 0.0005\end{array}$ & $\begin{array}{l}0.37 \\
\pm 0.16\end{array}$ & $\begin{array}{l}2.758 \\
\pm 0.006\end{array}$ & $\begin{array}{l}0.0033 \\
\pm 0.0016\end{array}$ \\
\hline
\end{tabular}

interatomic distance $0.23 \pm 0.01 \AA$ larger than that for the In-P bonds. The increased In-In bond length is in agreement with previous studies of InP amorphised by dominant nuclear energy loss $[3,4]$. The total coordination number for In atoms yields $3.73 \pm 0.41$. This is slightly less than the values stated by Glover et al. [3] and Azevedo et al. [4], who give $4.16 \pm 0.32$ and $3.90 \pm 0.30$, respectively. A similar behaviour follows for the percentage of "wrong" bonds which is reported to be $14 \%$ and $18 \%$, respectively, compared to $10 \%$ for the present study.

Thus, the general features found for samples amorphised by irradiation with a dominant nuclear energy loss can also be observed for a SHI induced amorphous phase. However, there are small differences in the values obtained for the structural parameters in particular the lower In-In coordination number and the unchanged In-P bond length. Whether these differences are due to a different short-range order as suggested by the report of Gaiduk et al. [10] or influenced by different experimental conditions cannot be determined unambiguously from this study. Hence, a comparative study of InP samples amorphised by irradiation with dominant nuclear or dominant electronic energy loss is currently underway.

\section{Summary}

Artifact free amorphous InP samples with the amorphous phase produced by electronic energy deposition were prepared for EXAFS measurements using $180 \mathrm{MeV} \mathrm{Au}^{13+}$ irradiation and a combination of semiconductor processing techniques and chemical etching. Analysis of the data clearly shows the amorphous state of the material, i.e. no coherent scattering contributions from beyond the first shell is observed. Furthermore, the Debye-Waller factor of the In-P scattering (first shell) is increased compared to the crystalline material. Both findings demonstrate the structural disorder introduced during the crystalline-to-amorphous transition. The data also shows that the first shell scattering peak of the amorphous phase comprises two different frequencies corresponding to In-P and In-In bonds. The homopolar coordinations represent chemical disorder in addition to the structural disorder mentioned above and are a characteristic feature of amorphous compound semiconductors. A comparative EXAFS study of the amorphous phase of InP produced by either dominant electronic or dominant nuclear energy deposition, now in progress, will yield further insight into the subtle differences observed.

\section{Acknowledgements}

The authors were supported by the Australian Synchrotron Research Program. P.K., A.P.B. and M.C.R. further acknowledge the support of the Australian Research Council.

\section{References}

[1] M.C. Ridgway, C.J. Glover, G.deM. Azevedo, S.M. Kluth, K.M. Yu, G.J. Foran, Nucl. Instr. and Meth. B 238 (2005) 294.

[2] E. Wendler, B. Breeger, Ch. Schubert, W. Wesch, Nucl. Instr. and Meth. B 147 (1999) 155.

[3] C.J. Glover, M.C. Ridgway, K.M. Yu, G.J. Foran, T.W. Lee, Y. Moon, E. Yoon, Appl. Phys. Lett. 74 (1999) 1713.

[4] G.deM. Azevedo, C.J. Glover, M.C. Ridgway, K.M. Yu, G.J. Foran, Phys. Rev. B 68 (2003) 115204.

[5] L.J. Lewis, A. de Vita, Phys. Rev. B 57 (1998) 1594.

[6] M. Toulemonde, C. Dufour, E. Paumier, Phys. Rev. B 46 (1992) 14362.

[7] O. Herre, W. Wesch, E. Wendler, P.I. Gaiduk, F.F. Komarov, S. Klaumünzer, P. Meier, Phys. Rev. B 58 (1998) 4832.

[8] W. Wesch, A. Kamarou, E. Wendler, Nucl. Instr. and Meth. B 225 (2004) 111.

[9] J.F. Ziegler, J.P. Biersack, U. Littmark, The Stopping and Range of Ions in Solids, Pergamon, New York, 2003.

[10] P.I. Gaiduk, F.F. Komarov, V.S. Tishkov, Phys. Rev. B 61 (2000) 15785.

[11] M. Newville, J. Synchrotron Radiat. 8 (2001) 322.

[12] B. Ravel, M. Newville, J. Synchrotron Radiat. 12 (2005) 537.

[13] J.J. Rehrs, R.C. Albers, Rev. Mod. Phys. 72 (2000) 621. 